\title{
Signature of an Intermediate-Mass Black Hole in the Central Molecular Zone of Our Galaxy
}

\author{
Tomoharu Oka, Reiko Mizuno, Kodai Miura and Shunya Takekawa
}

School of Fundamental Science and Technology, Graduate School of Science and Technology, Keio University, 3-14-1 Hiyoshi, Yokohama, Kanagawa 223-8522, Japan

email: tomo@phys.keio.ac.jp

\begin{abstract}
The high-velocity compact cloud CO-0.40-0.22 was mapped in 22 molecular lines with the NRO $45 \mathrm{~m}$ radio telescope and the ASTE $10 \mathrm{~m}$ telescope. The map of each detected line shows that this cloud has a compact appearance $(d \simeq 3 \mathrm{pc})$ and extremely broad velocity width $\left(\Delta V \simeq 100 \mathrm{~km} \mathrm{~s}^{-1}\right)$. The representative position-velocity map along the major axis shows that CO- $0.40-0.22$ consists of an intense region with a shallow velocity gradient and a less intense high-velocity wing. This kinematical structure can be attributed to a gravitational kick to the molecular cloud caused by an invisible compact object with a mass of $\sim 10^{5} M_{\odot}$. Its compactness and the absence of a counterpart at other wavelengths suggest that this massive object is an intermediate-mass black hole.
\end{abstract}

Keywords. galaxies: nuclei — Galaxy: center — ISM: clouds - ISM: molecules

\section{Introduction}

Most galaxies, including the Milky Way, are thought to have black holes (BHs) with masses greater than a million solar masses $\left(M_{\odot}\right)$ at their centers. However, the origins of such supermassive black holes (SMBHs) remain unknown. One possible scenario is that black holes with masses of $\sim 10^{3} M_{\odot}$, which are formed by runaway coalescence of stars in young compact star clusters (Portegies Zwart et al. 1999), merge at the center of a galaxy to form an SMBH (Ebisuzaki et al. 2001). To confirm the merging scenario, the ability to unambiguously detect intermediate-mass black holes (IMBHs; $M=10^{2-5} M_{\odot}$ ) is essential. Many candidates for IMBHs have been proposed to date on the basis of their ultraluminous nature (Fabbiano 2006; Roberts 2007), low-temperature blackbody spectral components (Miller et al. 2003), and quasi-periodic oscillations (Casella et al. 2008). However, none of these IMBH candidates are widely accepted as definitive, thus their existence is a long-standing controversy.

Recently, while investigating CO $J=3-2$ survey data of the Galactic CMZ obtained with the Atacama Submillimeter Telescope Experiment (ASTE) $10 \mathrm{~m}$ telescope (Oka et al. 2012), we noticed a peculiar molecular cloud at galactic longitude $-0.40^{\circ}$ and galactic latitude $-0.22^{\circ}$, with local standard of rest (LSR) velocities ranging from -120 to -20 $\mathrm{km} \mathrm{s}^{-1}$. This CO-0.40-0.22 is a compact $(<5 \mathrm{pc})$ cloud with an extremely broad velocity width $\left(\sim 100 \mathrm{~km} \mathrm{~s}^{-1}\right)$ and a very high $\mathrm{CO} J=3-2 / J=1-0$ intensity ratio $(\geqslant 1.5$; Fig.1). The high ratio implies that the cloud consists of dense, warm, and moderate opacity gas. It belongs to a peculiar category of molecular clouds, namely, high-velocity compact clouds (HVCCs; e.g., Oka et al. 1998; 1999; 2012), which were originally identified in the CO $J=1-0$ emission survey data (Oka et al. 1998). The position of CO-0.40-0.22 is approximately $0.2^{\circ}$ Galactic southeast of the massive star-forming region Sgr C, being displaced by $\sim 60 \mathrm{pc}$ in projected distance from the Galactic nucleus. 


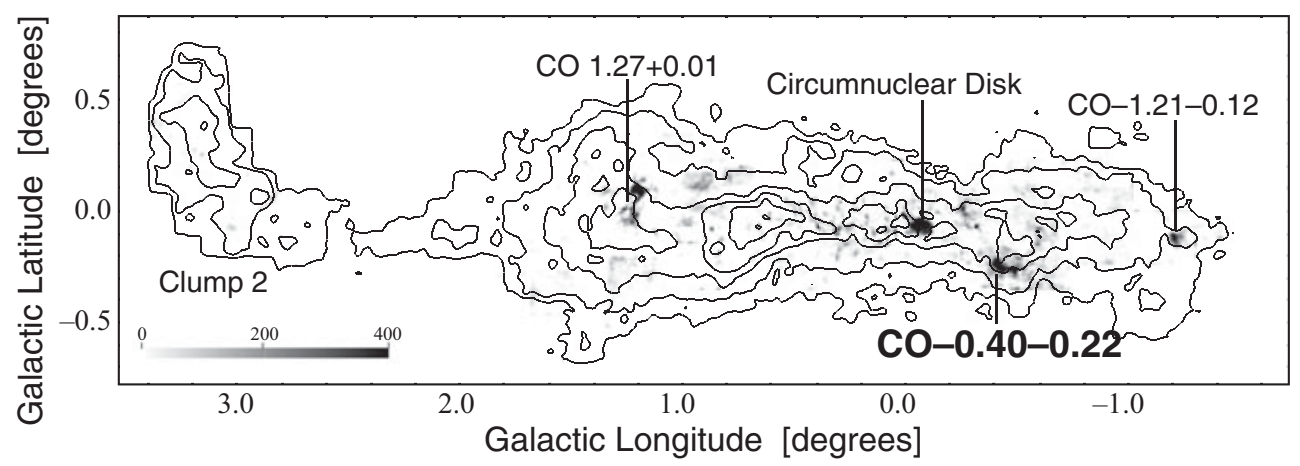

Figure 1. Map of the CO $J=3-2$ emission integrated for data with a high $\mathrm{CO} J=3-2 / J=1-0$ intensity ratio $(\geqslant 1.5)$. Contours show the velocity-integrated intensity of $\mathrm{CO} J=1-0$ emission.

\section{NRO 45m and ASTE Observations}

We performed mapping observations of $\mathrm{CO}-0.40-0.22$ in 22 molecular lines using the Nobeyama Radio Observatory (NRO) $45 \mathrm{~m}$ radio telescope and the ASTE $10 \mathrm{~m}$ telescope (see Oka et al. 2016 for details). We observed the $3^{\prime} \times 3^{\prime}$ area around $\mathrm{CO}-0.40-0.22$ in the on-the-fly mapping mode. The half-power beamwidth of the NRO $45 \mathrm{~m}$ telescope is $\simeq 20^{\prime \prime}$ at $86 \mathrm{GHz}$, while that of the ASTE is $\simeq 22^{\prime \prime}$ at $350 \mathrm{GHz}$. The obtained data were reduced using the NOSTAR reduction package. We subtracted the baselines of all spectra by fitting first- or third-order polynomial lines. We scaled the antenna temperature by multiplying it by $1 / \eta_{\mathrm{MB}}$ to obtain the main-beam temperature, $T_{\mathrm{MB}}$. All the data were resampled onto a $7^{\prime \prime} .5 \times 7^{\prime \prime} 5 \times 2 \mathrm{~km} \mathrm{~s}^{-1}$ grid to obtain the final maps.

\section{Kinematics and Energetics}

Nineteen of the 22 lines were detected from the center of CO-0.40-0.22. All the detected lines show that CO-0.40-0.22 has a compact appearance. The broad velocity width nature $\left(\sigma_{\mathrm{V}}>20 \mathrm{~km} \mathrm{~s}^{-1}\right)$ is also common. We present the velocity-integrated map and a positionvelocity map of the $\mathrm{SiO} J=2-1$ line, as it represents the spatial velocity structure of $\mathrm{CO}-$ 0.40-0.22 (Fig.2). It consists of an intense component with a shallow velocity gradient and a less intense high-velocity wing. Shock probe lines, such as the $\mathrm{SiO}, \mathrm{SO}, \mathrm{CH}_{3} \mathrm{OH}$, and HCN $J=4-3$ lines, commonly show this behavior. CO-0.40-0.22 is characterized by a rather featureless spatial velocity structure. This is in sharp contrast with CO 0.02-0.02 (Oka et al. 1999; 2008) or CO 1.27+0.01 (Oka et al. 2001; Tanaka et al. 2007), which are HVCCs containing expanding shells or emission cavities.

A gas mass of $M_{\text {gas }}=10^{3.6} M_{\odot}$ is derived from the HCN $J=1-0$ line intensity using the large velocity gradient model assuming $T_{\mathrm{k}}=60 \mathrm{~K}, n\left(\mathrm{H}_{2}\right) \geqslant 10^{6.5} \mathrm{~cm}^{-3}$, and $[\mathrm{HCN}] /\left[\mathrm{H}_{2}\right]=$ $10^{-7.3}$. A size parameter of $1.0 \mathrm{pc}$ and velocity dispersion of $20 \mathrm{~km} \mathrm{~s}^{-1}$ give a virial theorem mass of $M_{\mathrm{VT}}=1 \times 10^{6} M_{\odot}$. This yields a very large virial parameter, $M_{\mathrm{VT}} / M_{\mathrm{gas}} \sim$ 300 , indicating that the gas mass is definitely insufficient to bind the cloud by its selfgravity. The kinetic energy amounts to $E_{\text {kin }}=10^{49.7}$ erg if the velocity dispersion is dominated by random motion. If it is expanding at $V_{\exp }=40 \mathrm{~km} \mathrm{~s}^{-1}$ (half of the velocity extent), the kinetic energy becomes $E_{\text {kin }}=10^{49.8} \mathrm{erg}$.

\section{Gravitational Kick Model}

The lack of counterparts of CO-0.40-0.22 at other wavelengths disfavors formation scenarios based on internal explosive events. Further, the absence of an expanding feature 

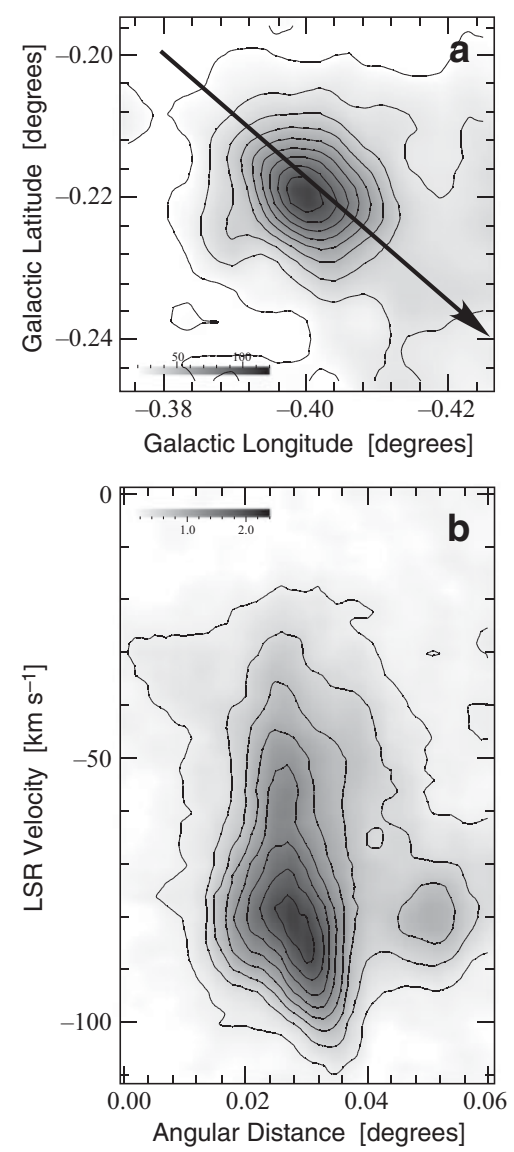

Figure 2. (a) Map of $\mathrm{SiO} J=2-1$ emission integrated over velocities from -110 to 0 $\mathrm{km} \mathrm{s}^{-1}$. A solid circle shows the half-power beamwidth (HPBW) of the telescope at 86 GHz. (b) Position-velocity map of $\mathrm{SiO} J=2-1$ emission along the thick arrow indicated in panel a. The horizontal axis indicates the position along the arrow.

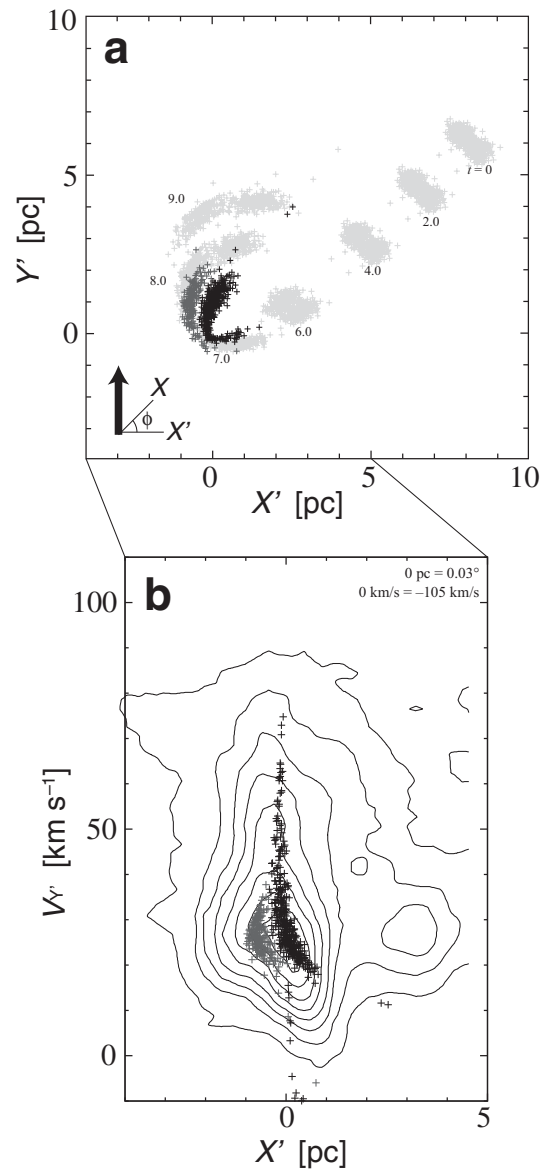

Figure 3. (a) Time evolution of two clouds in the orbital plane. Thick arrow indicates the line-of-sight direction. (b) Position-velocity plot of the simulated clouds superposed on the $\mathrm{SiO} J=2-1$ map. The vertical axis shows the line-of-sight velocity, and the horizontal axis shows the transverse offset from the mass point.

or a cavity also reduces support for internal explosion/outflow scenarios. A promising candidate for an explanation of the formation scenario involves a "gravitational kick" to a small incoming cloud by a compact source. For example, a point-like mass of $10^{5}$ $M_{\odot}$ can accelerate a cloud coming from infinity to $\sim 90 \mathrm{~km} \mathrm{~s}^{-1}$ at a distance of $0.1 \mathrm{pc}$. This scenario can easily explain the compact appearance and very large velocity width of $\mathrm{CO}-0.40-0.22$.

For a semiquantitative comparison, we simulated the position-velocity behavior of an incoming cloud. We placed a cloud of 200 test particles with a $\sigma=0.2$ pc Gaussian centered at $\sim 10 \mathrm{pc}$ away from the massive object. The initial velocity was set to $10 \mathrm{~km} \mathrm{~s}^{-1}$. After a number of trials, we found that the parameter set which reproduces the positionvelocity behavior of CO-0.40-0.22 well (Fig.2). These simulations indicate that the mass of the point-like gravitational source should be $\sim 10^{5} M_{\odot}$. The locus of the gravitational 
source corresponds to the center of $\mathrm{CO}-0.40-0.22$, from which the positive-side highest velocity emission arises.

\section{Signature of an Intermediate-Mass Black Hole?}

Supposing the gravitational kick scenario is valid, the size of the object must be significantly smaller than $0.1 \mathrm{pc}$ (pericenter distance). The inferred mass and size correspond to an average mass density of $\rho \simeq 2 \times 10^{7} M_{\odot} \mathrm{pc}^{-3}$. This mass density is comparable to that of the core of M15, which is one of the most densely packed (core-collapsed) globular clusters in the Milky Way Galaxy (Djorgovski \& King 1984). The lack of counterparts at other wavelengths is inconsistent with the massive stellar cluster interpretation, unless the cluster consists almost entirely of dark stellar remnants, such as neutron stars and BHs. Therefore, it is most likely that the massive compact object responsible for the formation of $\mathrm{CO}-0.40-0.22$ is an IMBH.

The origin of such a "massive" IMBH is controversial. The relation between the BH mass and the stellar system mass (e.g., Kormendy \& Ho 2013) indicates that a $10^{5} M_{\odot}$ BH may be involved in a stellar system of $\sim 10^{8} M_{\odot}$, which falls into the mass range of dwarf galaxies. Recently, SMBHs have been found at the centers of dwarf galaxies (Reines et al. 2011; Seth et al. 2014) and, in the vicinity of the Milky Way Galaxy, over 20 dwarf satellite galaxies have been discovered to date. It is believed that large galaxies such as the Milky Way have grown to their present form by cannibalizing their smaller neighbors. Thus, it is natural to suggest that the $10^{5} M_{\odot} \mathrm{BH}$ in the Galactic $\mathrm{CMZ}$ was the nucleus of a cannibalized dwarf galaxy.

Some of the HVCCs in the Galactic CMZ (Oka et al. 1998; 1999; 2012; Tanaka et al. 2007; 2014) also provide possible candidates for nonluminous BHs. High-resolution aperture synthesis imaging of such HVCCs will reveal their detailed spatial structures and kinematics, which are crucial to confirming the presence of BHs within them. The detection of such compact high-velocity features in nearby galaxies will increase the number of IMBH candidates and thereby generalize our gravitational kick interpretation.

\section{References}

Casella, P., Ponti, G., Patruno, A., et al. 2008, MNRAS, 387, 1707

Djorgovski, S. \& King, I. R. 1984, ApJ, 277, L49

Ebisuzaki, T., Makino, J., Tsuru, T. G., et al. 2001, ApJ, 562, L19

Fabbiano, G. 2006, ARAE $A, 44,323$

Kormendy, J. \& Ho, L. C. 2013, ARA\&A, 51, 511

Miller, J. M., Fabbiano, G., Miller, M. C., \& Fabian, A. C. 2003, ApJ, 585, L37

Oka, T., Hasegawa, T., Sato, F., Tsuboi, M., \& Miyazaki, A. 1998, ApJS, 118, 455

Oka, T., White, G. J., Hasegawa, T., Sato, F., Tsuboi, M., \& Miyazaki, A. 1999, ApJ, 515, 249

Oka, T., Hasegawa, T., Sato, F., Tsuboi, M., \& Miyazaki, A. 2001, PASJ, 53, 787

Oka, T., Nagai, M., Kamegai, K., Tanaka, K., \& Kuboi, N. 2007, PASJ, 59, 15

Oka, T., Hasegawa, T., White, G. J., Sato, F., Tsuboi, M., \& Miyazaki, A. 2008, PASJ, 60, 429

Oka, T., Onodera, Y., Nagai, M., et al. 2012, ApJS, 201, 14

Oka, T., Mizuno, R., Miura, K., \& Takekawa, S. 2016, ApJ, 816, L7

Portegies Zwart, S. F., Makino, J., McMillan, S. L. W., \& Hut, P. 1999, ApJ, 348, 117

Reines, A. E., Sivakoff, G. R., Johnson, K. E., \& Brogan, C. L. 2011, Nature, 470, 66

Roberts, T. P. 2007, ApESS , 311, 203

Tanaka K., Kamegai, K., Nagai, M., \& Oka, T. 2007, PASJ, 59, 323

Tanaka, K., Oka, T., Matsumura, S., Nagai, M., \& Kamegai, K. 2014, ApJ, 737, 62

Seth, A. C., van den Bosch, R., Mieske, S., et al. 2014, Nature, 513, 398 